\title{
Composição da ictiofauna em função da fisiografia de um riacho costeiro de Floresta Atlântica - Brasil
}

\author{
Ana Tereza Bittencourt Guimarães ${ }^{1,3}$, Márcia Santos de Menezes ${ }^{2}$ \& Alberto Carvalho Peret ${ }^{1}$ \\ ${ }^{1}$ Programa de Pós-graduação em Ecologia e Recursos Naturais, Centro de Ciências Biológicas e da Saúde, \\ Universidade Federal de São Carlos - UFSCar, CP 676, CEP 13565-905, São Carlos, SP, Brasil \\ ${ }^{2}$ Laboratório de Ecologia de Rios, Setor de Ciências Biológicas, Universidade Federal do Paraná - UFPR, \\ CP 19031, CEP 81531-980, Curitiba, PR, Brasil \\ ${ }^{3}$ Autor para correspondência: Ana Tereza Bittencourt Guimarães, e-mail: anat@brturbo.com.br
}

BITTENCOURT GUIMARÃES, A.T., MENEZES, M.S. \& PERET, A.C. Ichthyofauna composition related to physiography in a coastal stream of Atlantic Forest, Brazil. Biota Neotrop. 10(2): http://www.biotaneotropica. org.br/v10n2/en/abstract?article+bn01010022010.

\begin{abstract}
In spite of the impacts that tropical streams are submitted, they still present a great fish diversity. The knowledge of the fish structure and composition is an important attempt in order to stabilish evaluation parameters that can favour conservation of these environments. The icthyofaunal composition related to physiographic features of coastal streams of south Brazil is an important contribution to the interpretation of these ecosystem dynamics. We perform fish collections in five sampling sites in the Pinto River, placed in Morretes municipality in the Paraná State, Brazil. The samples were accomplished between May 2006 and November 2007, using electric fishing along $50 \mathrm{~m} /$ hour in each site. The collected animals were fixed in formaline $10 \%$. Each site (point) was classified in relation to the bottom substratum, depth, width average, and current speed. Direct relationship between substratum type and fish compostition was observed. Siluriformes were predominant in places with coarseness granulometry, such as boulders, cobbles and pebbles. On the other hand, Characiformes were predominant in places with smaller granulometry, such as coarse sand and gravel.
\end{abstract}

Keywords: Siluriformes, Characiformes, longitudinal distribution, coastal streams.

BITTENCOURT GUimarÃES, A.T., MENEZES, M.S. \& PERET, A.C. Composição da ictiofauna em função da fisiografia de um riacho costeiro de Floresta Atlântica - Brasil. Biota Neotrop. 10(2): http://www. biotaneotropica.org.br/v10n2/pt/abstract?article+bn01010022010.

Resumo: Os riachos costeiros apresentam grande diversidade de peixes, apesar dos impactos a que são submetidos. O conhecimento da estrutura e da composição da ictiofauna constitui importante esforço para levantamento de parâmetros de avaliação que possam proporcionar atitudes para a conservação destes ambientes. A composição da ictiofauna relacionada a características fisiográficas de riachos costeiros do sul do Brasil constitui-se uma contribuição para a interpretação da dinâmica destes ecossistemas. Foram realizadas coletas de peixes em cinco pontos amostrais do Rio do Pinto, localizado no município de Morretes, Paraná, Brasil. As coletas foram realizadas entre os meses de maio de 2006 a novembro de 2007, utilizando a técnica de pesca elétrica, com um esforço amostral de $50 \mathrm{~m}$ /hora em cada localidade. Os animais coletados foram fixados em formaldeído $10 \%$. Cada localidade foi classificada em relação ao tipo de substrato, profundidade, velocidade de correnteza e largura média. Foi possível observar a relação direta entre o tipo de substrato e a composição da ictiofauna. Em regiões onde a granulometria era maior, tais como matacões e seixos, prevaleceram os Siluriformes. Por outro lado, em regiões onde o sedimento apresentava menor granulometria, tais como areia grossa associada a cascalho, ocorreram espécies de Characiformes.

Palavras-chave: Siluriformes, Characiformes, substratos, distribuição longitudinal. 


\section{Introdução}

Muitas pesquisas têm sido concentradas na tentativa de compreender os processos ecológicos que ocorrem em riachos costeiros da Floresta Atlântica. A restauração e a conservação de ecossistemas como este, não pode prescindir da idealização de planos de avaliação da higidez dos sistemas por meio da valoração das características hidromorfológicas, químicas e biológicas (Tejerina-Garro et al. 2005).

A dinâmica dos riachos costeiros é diretamente afetada por eventos naturais. Aranha (2000) demonstrou como a diversidade da ictiofauna é desestabilizada em função da ocorrência de trombas d'água, alterando-se a dominância das espécies ocorrentes. Ferreira (2007) conseguiu demonstrar como a variação da vegetação ripária pode influenciar na ocupação das espécies de peixes, havendo a alteração dos tipos ecomorfológicos, bem como dos hábitos alimentares. Entre estes autores, muitos outros abordam questões semelhantes sobre a influência de eventos estocásticos sobre a diversidade animal e vegetal (Grossman et al. 1982, Yant et al. 1984, Mathews 1986, Griffin et al. 2009).

Variáveis explicativas da ocorrência e distribuição das espécies de peixes em um córrego e/ou riacho podem estar relacionadas à fisiografia local, ilustrando tal fato em função do tipo de substrato, presença ou ausência de vegetação ripária submersa e velocidade de corrente (Ferreira \& Casatti 2006, Ferreira 2007). Silveira (2004) afirma que a textura do substrato, seu tamanho, níveis de compressão e espaço intersticial são importantes no movimento e estabelecimento de espécies de macroinvertebrados em um local. Considerando estes organismos como fonte alimentar para uma série de espécies de peixes, sua presença e distribuição podem influenciar na ocorrência e distribuição da ictiofauna. Contudo, não só a fisiografia é explicativa para a ocorrência das espécies, havendo também a influência de variáveis biológicas (Esteves \& Lobón-Cerviá 2001), físicas e químicas (Friberg et al. 2009).

No Brasil, ainda são poucos os trabalhos que realizaram o levantamento das características de riachos costeiros. Ainda são mais freqüentes os trabalhos que fazem abordagens biológicas, tais como levantamentos de ocorrência de espécies (Casatti et al. 2001, Barreto \& Aranha 2005, Ferreira 2007), comportamento alimentar (Aranha et al. 1998, Esteves \& Lobón-Cerviá 2001, Casatti 2002, Ferreira 2007), aspectos reprodutivos (Menezes et al. 2000, Ferreira 2007) e avaliações de integridade biológica (Araújo 1998, Casatti 2004).

Os fragmentos de Floresta Atlântica remanescentes no Brasil necessitam de mais estudos básicos que gerem subsídios teóricos para futuros trabalhos de restauração ambiental. O conhecimento sobre a composição e a caracterização das taxocenoses nos riachos costeiros do Paraná ainda é insuficiente e tem se tornado de especial importância, principalmente para a compreensão dos processos ecológicos. Desta maneira, a conduta científica não se refere apenas à preservação, mas também à reabilitação da estrutura ecológica, das funções e da integridade dos ambientes. A valoração de um ecossistema, bem como a formulação de modelos de avaliação de impacto ambiental, utiliza informações sobre biologia de espécies de peixes, sendo tal fato evidenciado por Lemes \& Garutti (2002) que afirmaram que riachos apresentam fisiografia plástica espacial e temporal, o que resulta em uma composição ictíica que explora o meio de acordo com habilidades e exigências biológicas.

Sendo assim, trabalhos sobre a biologia, a composição e a distribuição de peixes, tornam-se informações de base para a posterior construção de modelos que possibilitem prognósticos sobre as alterações ambientais provindas do impacto antrópico. Sendo assim, os objetivos deste trabalho foram:
1) avaliar a composição e a distribuição da ictiofauna em um riacho costeiro de Floresta Atlântica;

2) relacionar a composição e a distribuição da ictiofauna com a estrutura fisiográfica de diferentes porções de um riacho costeiro; e

3) demonstrar as estratégias ecomorfológicas para adaptação em diferentes estruturas fisiográficas de um riacho costeiro.

\section{Material e Métodos}

Coletas de peixes foram realizadas mensalmente durante o período compreendido entre maio de 2006 a novembro de 2007. As amostragens foram realizadas em cinco pontos na microbacia do Rio do Pinto, situados na Serra do Mar, município de Morretes, Paraná, sul do Brasil. O primeiro localiza-se no Rio Caiuru (P1 $25^{\circ} 34.356^{\prime} \mathrm{S}$ e $\left.48^{\circ} 53.008^{\prime} \mathrm{O}\right)$. Os demais se localizam no Rio do

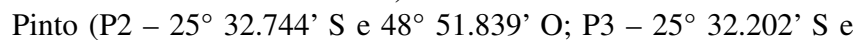
$48^{\circ} 50.831^{\prime}$ O; $\mathrm{P} 4-25^{\circ} 30.282^{\prime} \mathrm{S}$ e $48^{\circ} 49.834^{\prime} \mathrm{O}, \mathrm{P} 5-25^{\circ} 29.948^{\prime} \mathrm{S}$ e $48^{\circ} 48.903$ ' O) (Figura 1). Todas as coletas foram realizadas apenas em dias com sol e/ou sem chuva.

$\mathrm{O}$ apetrecho de coleta utilizado foi a pesca elétrica, com um esforço amostral de $50 \mathrm{~m} /$ hora em cada localidade. Os animais coletados foram fixados em Formaldeído $10 \%$ e após 48 horas transferidos para álcool $70^{\circ}$. As espécies de peixes coletadas foram identificadas até o menor nível taxonômico possível por meio de chaves de identificação específicas. Exemplares testemunhos foram depositados na Coleção de Peixes do Laboratório de Ecologia de Rios do Departamento de Zoologia da Universidade Federal do Paraná.

Em cada um dos pontos de coleta foi registrado o tipo de corrente, conforme determinado por Barreto \& Aranha (2005), sendo considerada correnteza ausente $0 \mathrm{~m} / \mathrm{s}$, lenta de 0,01 a $0,25 \mathrm{~m} / \mathrm{s}$, moderada de 0,26 a $0,50 \mathrm{~m} / \mathrm{s}$, rápida de 0,51 a $0,75 \mathrm{~m} / \mathrm{s}$ e torrencial acima de $0,76 \mathrm{~m} / \mathrm{s}$.

A granulometria do cascalho foi definida em cada ponto de coleta, de acordo com Teixeira et al. (2000), sendo denominado de matacão os sedimentos com intervalo granulométrico superior a $256 \mathrm{~mm}$; bloco os que apresentam variação entre 64 a $256 \mathrm{~mm}$; seixo com 4 a $64 \mathrm{~mm}$ e grânulo de 2 a $4 \mathrm{~mm}$.

Para cada espécie foi calculado mensalmente o grau de importância relativa, pela freqüência de ocorrência percentual (FO\%), e sua percentagem numérica (PN\%), conforme Loebmann \& Vieira (2005). Desta maneira, foi possível identificar as espécies como:

a) freqüentes e abundantes, sendo aquelas que ultrapassaram as médias de $\mathrm{PN} \%$ e $\mathrm{FO} \%$;

b) freqüentes e não abundantes, aquelas que não ultrapassam as médias de PN\%, mas ultrapassam de FO\%; e

c) ocasionais, aquelas que não ultrapassaram as médias de PN\% e $\mathrm{FO} \%$.

As espécies foram classificadas como adaptadas para os ambientes de corredeira ou remansos quando apresentavam características ecomorfológicas específicas para tais ambientes, como por exemplo compressão dorso-ventral, nadadeiras peitorais amplas e estruturas modificadas para fixação espécies de ambientes de corredeiras; e compressão lateral, nadadeiras caudais amplas e boca terminal para espécies de ambientes com águas mais calmas. Estas características foram propostas por Watson \& Balon (1984). As diversidades foram calculadas por meio do índice de Equitabilidade de Pielou e comparadas por meio do teste t para diversidade, utilizando um nível de significância de 0,05 (Zar 1998). A similaridade entre os ambientes foi calculada por meio do índice de Similaridade de Bray-Curtis e posteriormente, expresso em dendrograma (Dajoz 2005). A significância do dendrograma foi analisada por meio do cálculo do valor cofenético. 


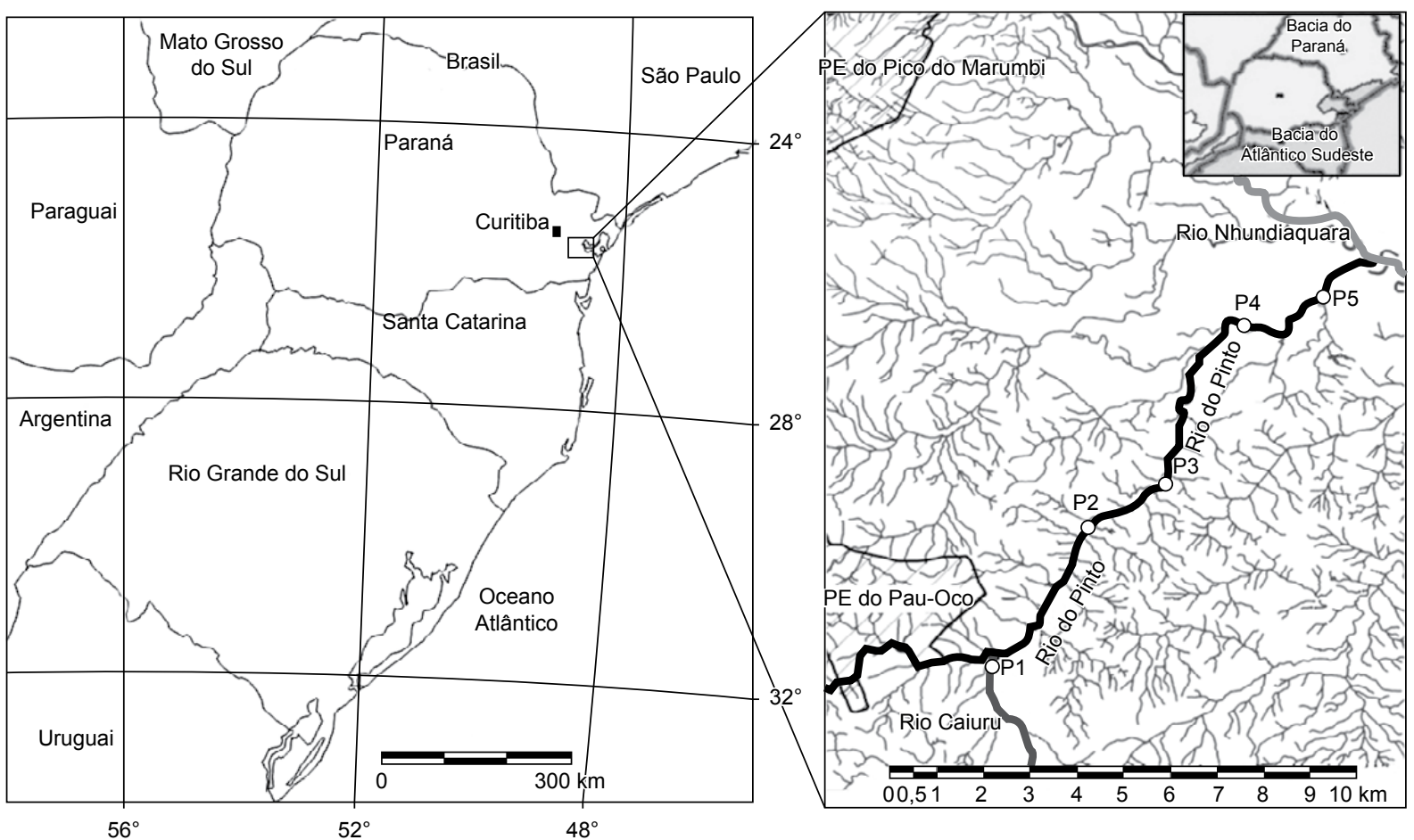

Figura 1. Mapa de localização dos pontos de Coleta. Ponto 1 (P1) situado no Rio Caiuru (destaque em cinza escuro); Ponto 2 (P2), Ponto 3 (P3), Ponto 4 (P4) e Ponto 5 (P5) situados no rio do Pinto (destaque em preto).

Figure 1. Location map of sampling sites. Site 1 (P1) placed in Caiuru River (dark ash); Site 2 (P2), Site 3 (P3), Site 4 (P4), and Site 5 (P5) placed in Pinto River (black).

Por fim, foi realizada a análise multivariada de correspondência (Hair et al., 1998) para verificar a associação da frequiência absoluta das espécies frequientes e abundantes com os pontos de coleta de acordo com os seus aspectos fisiográficos. Todos os testes estatísticos foram realizados no programa XLStat2009 (Addinsoft 2009).

\section{Resultados}

A microbacia do Rio do Pinto apresenta atividade antrópica crescente da nascente à foz. O ponto $\mathrm{P} 1$ está localizado no Rio Caiuru, que é um afluente do Rio do Pinto. Caracteriza-se por estar a $169 \mathrm{~m}$ de altitude em relação ao nível do mar, apresentando a correnteza do tipo torrencial $(0,95 \mathrm{~m} / \mathrm{s})$ e o substrato composto por grande quantidade de matacões e blocos. A profundidade variou entre 0,2 e $0,4 \mathrm{~m}$ e largura média de $6,8 \mathrm{~m}$. Nesta localidade, um trecho do leito está cimentado e parte das margens estão canalizadas com muretas para suportar a passagem de um oleoduto sob o leito do rio (Figura 2).

$\mathrm{O}$ ponto $\mathrm{P} 2$ distancia-se $3,9 \mathrm{~km}$ de $\mathrm{P} 1$ e localiza-se no Rio do Pinto. Apresenta-se a $73 \mathrm{~m}$ de altitude, com corrente caracterizada como rápida $(0,53 \mathrm{~m} / \mathrm{s})$. A profundidade variou entre 0,14 e $0,60 \mathrm{~m}$ e a largura média foi de $15 \mathrm{~m}$. O leito do rio é composto principalmente por matacões, blocos e seixos, apresentando sua vazão fluvial regulada pelas precipitações locais, as quais determinam a variação de correnteza. A vegetação ripária é composta por integrantes da família Poaceae, além de pequenas plantações de subsistência de banana, palmito, cana de açúcar e mandioca (Figura 3).

$\mathrm{O}$ ponto $\mathrm{P} 3$ distancia-se $2,2 \mathrm{~km}$ do $\mathrm{P} 2$, encontrando-se a $44 \mathrm{~m}$ de altitude e com corrente do tipo moderada $(0,37 \mathrm{~m} / \mathrm{s})$. A profundidade variou entre 0,33 e $0,48 \mathrm{~m}$, e sua largura atingiu $20 \mathrm{~m}$. Seu leito é semelhante ao $\mathrm{P} 2$, havendo a predominância de blocos. A vegetação é semelhante a observada no ponto $\mathrm{P} 2$, sendo que próximo às margens

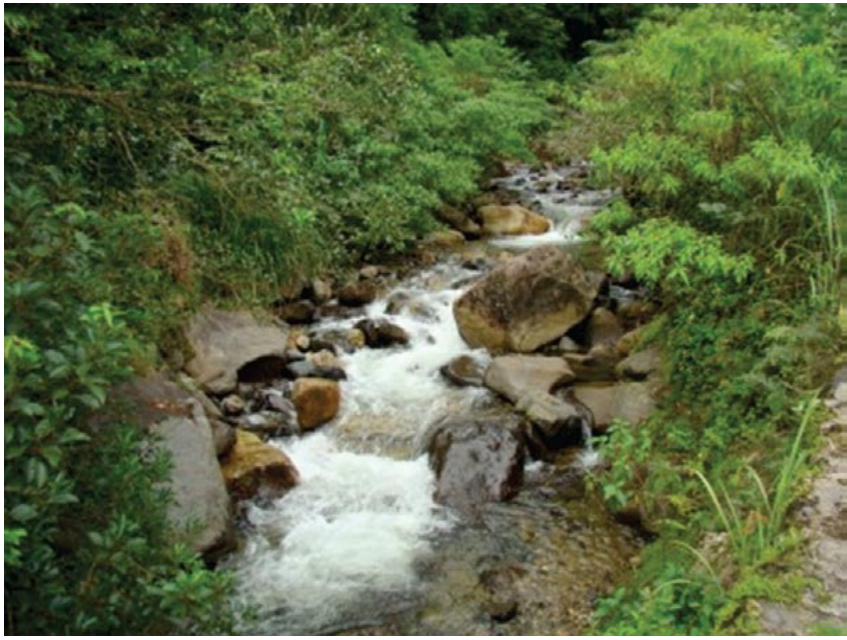

Figura 2. Ponto 1: Situado no Rio Caiuru, município de Morretes, PR. Profundidade máxima: 0,4 m; Correnteza: torrencial; Substrato: matacões e blocos. Vista à montante. Foto obtida em maio/2009.

Figure 2. Site 1: Placed in the Caiuru River, Morretes municipality in the Parana State. Maximum depth: 0.4 m; Current: torrential; Substrate: boulders and cobbles. Upper view. Photo taked on 2009/May.

também são observados cultivos de subsistência familiar e comerciais, tais como banana, palmito, chuchu e berinjela (Figura 4).

$\mathrm{O}$ ponto $\mathrm{P} 4$ encontra-se distante $4,5 \mathrm{~km}$ do $\mathrm{P} 3$. Localiza-se a $16 \mathrm{~m}$ de altitude, com porções onde a corrente é do tipo torrencial $(1,06 \mathrm{~m} / \mathrm{s})$ e grande parte de sua área com corrente rápida $(0,63 \mathrm{~m} / \mathrm{s})$. A profundidade observada variou de 0,10 a $0,80 \mathrm{~m}$, com largura 
média de $22 \mathrm{~m}$. Seu leito é composto principalmente por seixos e grânulos. Nesta região observou-se uma grande influência de dejetos residenciais e agrícolas provindos da população rural do município de Morretes. As áreas próximas à microbacia do rio nesta localidade são mais planas, com plantações comerciais de chuchu, maracujá e arroz (Figura 5).

$O$ ponto $\mathrm{P} 5$ distancia-se $2,2 \mathrm{~km}$ do $\mathrm{P} 4$, estando a $6 \mathrm{~m}$ de altitude. Sua corrente caracteriza-se como rápida $(0,74 \mathrm{~m} / \mathrm{s})$, com profundidade variável entre 0,2 e $1,5 \mathrm{~m}$. A largura média foi de $15 \mathrm{~m}$ e o leito é composto principalmente por grânulos e areia de diferentes intervalos granulométricos. A água apresenta odor desagradável devido à existência de dejetos residenciais. Cultivos de subsistências, tais como cana de açúcar, mandioca e banana, e grande quantidade de plantações comerciais de hortaliças e verduras são encontradas no entorno (Figura 6).

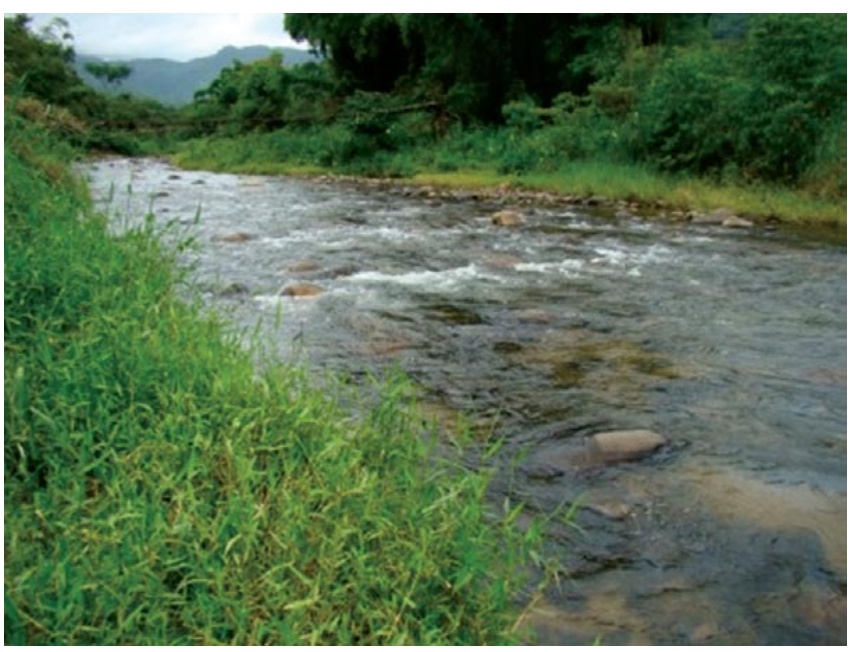

Figura 3. Pontos 2: Situado no Rio do Pinto, município de Morretes, PR. Profundidade máxima: $0,5 \mathrm{~m}$; Correnteza: rápida; Substrato: matacões, blocos e seixos. Vista à montante. Foto obtida em maio/2009.

Figure 3. Site 2: Placed in the Pinto River, Morretes municipality in the Parana State. Maximum depth: 0.5 m; Current: fast; Substrate: boulders, cobbles and pebbles. Upper view. Photo taked on 2009/May.

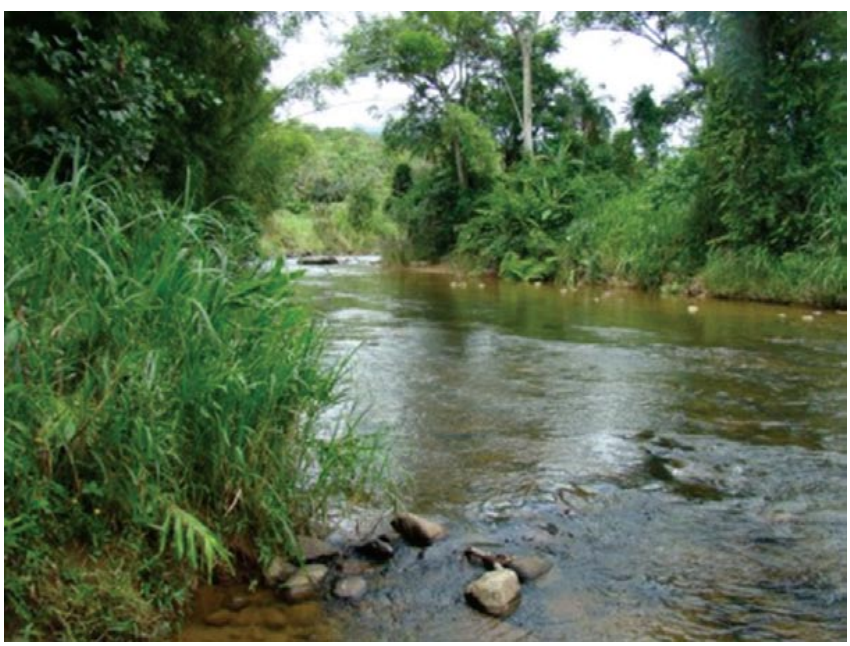

Figura 4. Ponto 3: Situado no Rio do Pinto, município de Morretes, PR. Profundidade máxima: 0,5 m; Correnteza: moderada (P3); Substrato: matacões, blocos e seixos. Vista à montante. Foto obtida em maio/2009.

Figure 4. Site 3: Placed in the Pinto River, Morretes municipality in the Parana State. Maximum depth: 0.5 m; Current: moderate; Substrate: boulders, cobbles and pebbles. Upper view. Photo taked on 2009/May.
Ao longo do trecho estudado, foram capturados 7116 exemplares de peixes, distribuídos em 12 famílias e 33 espécies, sendo as ordens Siluriformes $(54,08 \%)$ e Characiformes $(39,66 \%)$ predominantes em FO\% e PN\%, seguidas de Perciformes (2,82\%), Cyprinodontiformes $(2,55 \%)$, Symbranchiformes $(0,53 \%)$ e Gymnodontiformes $(0,35 \%)$ (Tabela 1). Os Siluriformes foram principalmente representados por Rineloricaria sp. (22\%) e Isbrueckerichthys duseni (19\%), os Characiformes por Charicidium pterostictum (35\%) e Characidium lanei (30\%), os Perciformes por Awaous tajasica (70\%), os Cyprinodontiformes por Phalloceros caudimaculatus (100\%), Symbranchiformes por Symbranchus marmoratus (100\%) e Gymnodontiformes por Gymnotus carapo (54\%) e Gymnotus panterinus $(46 \%)$.

O ponto $\mathrm{P} 1$ mostrou-se o menos rico, contendo apenas 16 espécies, seguido do P5 (22 espécies), P3 (24 espécies), P2 (27 espécies) e

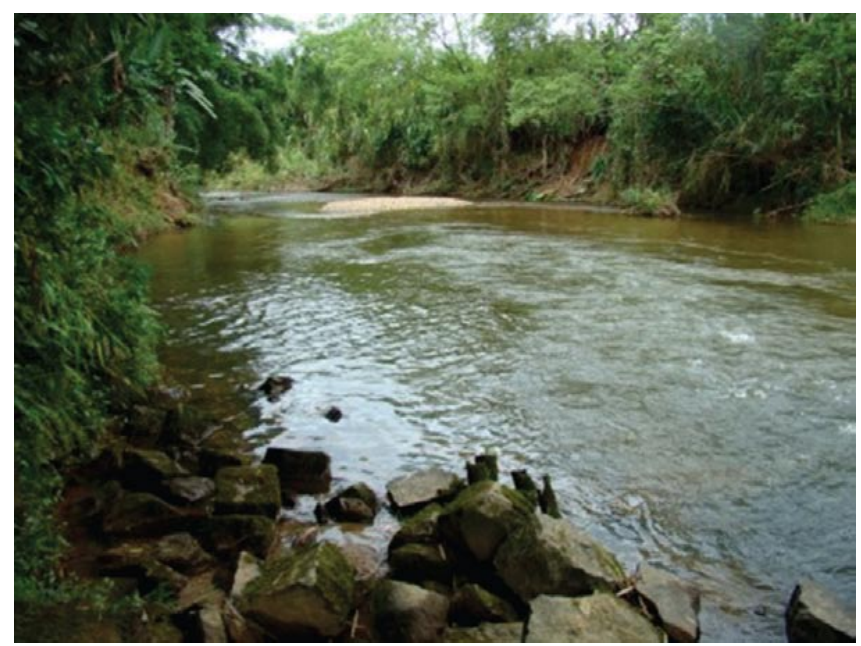

Figura 5. Ponto 4: Situado no Rio do Pinto, município de Morretes, PR. Profundidade máxima: 0,4 m; Correnteza: rápida à torrencial; Substrato: seixos e grânulos. Vista à montante. Foto obtida em maio/2009.

Figure 5. Site 4: Placed in the Pinto River, Morretes municipality in the Parana State. Maximum depth: $0.4 \mathrm{~m}$; Current: fast to torrential; Substrate: pebbles and gravel. Upper view. Photo taked on 2009/May.

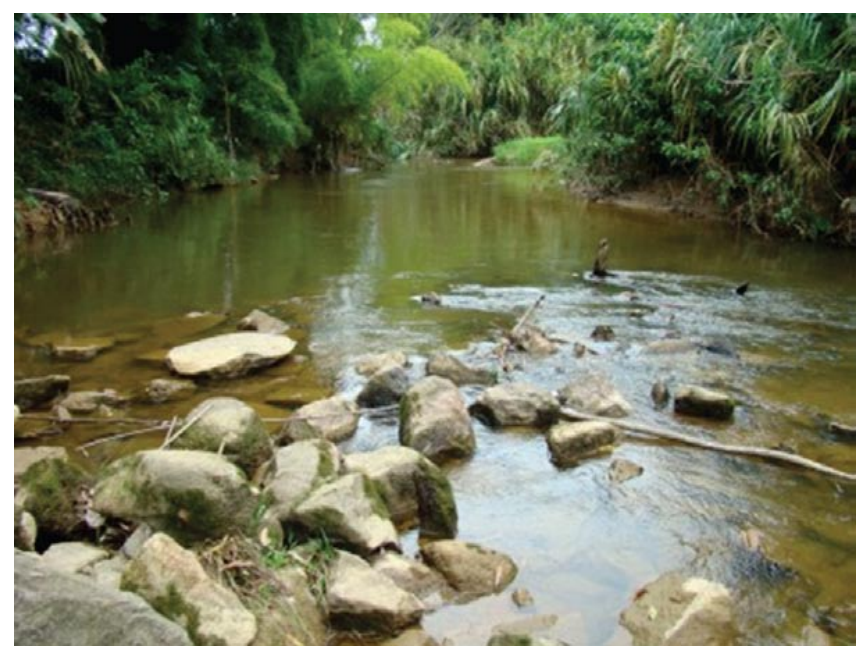

Figura 6. Ponto 5: Situado no Rio do Pinto, município de Morretes, PR. Profundidade máxima: 1,5 m; Correnteza: rápida; Substrato: grânulos e areia. Vista à montante. Foto obtida em maio/2009.

Figure 6. Site 5: Placed in the Pinto River, Morretes municipality in the Parana State. Maximum depth: $1.5 \mathrm{~m}$; Current: fast; Substrate: gravel and sand. Upper view. Photo taked on 2009/May. 
Composição da ictiofauna em função da fisiografia...

Tabela 1. Relação das espécies de peixes coletados no Rio do Pinto, Morretes, Paraná.

Table 1. Relationship of the species of fish collected in the River Pinto, Morretes, Parana.

\begin{tabular}{|c|c|c|c|}
\hline Ordem & Família & Subfamília & Espécie \\
\hline \multirow[t]{2}{*}{ Gymnodontiformes } & GYMNOTIDAE & & Gymnotus carapo (Linnaeus, 1758) \\
\hline & & & Gymnotus pantherinus (Steindachner, 1908) \\
\hline Symbranchiformes & SYMBRANCHIDAE & & Synbranchus marmoratus (Bloch, 1795) \\
\hline Cyprinodontiformes & POECILIIDAE & & Phalloceros caudimaculatus (Hensel, 1868) \\
\hline \multirow[t]{2}{*}{ Perciformes } & GOBIIDAE & Gobionellinae & Awaous tajasica (Lichtenstein, 1822) \\
\hline & CICHLIDAE & Cichlinae & $\begin{array}{l}\text { Crenicichla lacustris (Castelnau, 1855) } \\
\text { Geophagus brasiliensis (Quoy \& Gaimard, 1824) }\end{array}$ \\
\hline \multirow[t]{9}{*}{ Characiformes } & CHARACIDAE & Glandulocaudinae & Astyanax sp. \\
\hline & & & Oligosarcus hepsetus (Cuvier, 1829) \\
\hline & & & Bryconamericus microcephalus (Miranda Ribeiro, 1908) \\
\hline & & & Deuterodon langei (Travassos, 1957) \\
\hline & & & Hollandichthys multifasciatus (Eigenmann \& Norris, 1900) \\
\hline & & & Mimagoniates microlepis (Steindachner, 1877) \\
\hline & CRENUCHIDAE & Characidiinae & Characidium lanei (Travassos, 1967) \\
\hline & & & Characidium pterostictum (Gomes, 1947) \\
\hline & ERYTHRINIDAE & & Hoplias malabaricus (Bloch, 1794) \\
\hline \multirow[t]{16}{*}{ Siluriformes } & CALLICHTHYDAE & Callichthyinae & $\begin{array}{l}\text { Scleromystax barbatus (Quoy \& Gaimard, 1824) } \\
\text { Hoplosternum littorale (Hancock, 1828) }\end{array}$ \\
\hline & LORICARIIDAE & Hypostominae & Ancistrus multispinis (Regan, 1912) \\
\hline & & & Pareiorhaphis splendens (Bizerril, 1995) \\
\hline & & & Kronichthys lacerta (Nichols, 1919) \\
\hline & & Neoplecostominae & Isbrueckerichthys duseni (Miranda Ribeiro, 1907) \\
\hline & & Hypoptomatinae & Pseudotothyris obtusa (Miranda Ribeiro, 1911) \\
\hline & & & Hisonotus leucofrenatus (Miranda Ribeiro, 1908) \\
\hline & & & Schizolecis güntheri (Miranda Ribeiro, 1918) \\
\hline & & Loricariinae & Rineloricaria sp. \\
\hline & HEPTAPTERIDAE & & Pimelodella pappenheimi (Ahl, 1925) \\
\hline & & & Acentronichthys leptos (Eigenmann \& Eigenmann, 1889) \\
\hline & & & Rhamdia quelen (Quoy \& Gaimard, 1824) \\
\hline & & & Rhamdioglanis transfasciatus (Miranda Ribeiro, 1908) \\
\hline & TRICHOMYCHTERIDAE & Trichomycterinae & Trichomycterus davisi (Haseman, 1911) \\
\hline & & Stegophilinae & Homodiaetus graciosa (Koch, 2002) \\
\hline & PSEUDOPIMELODIDAE & & Microglanis cottoides (Boulenger, 1891) \\
\hline
\end{tabular}

P4 (28 espécies). A menor riqueza do ponto P1 reflete os valores de diversidade, sendo o único ponto que difere quanto à composição da comunidade em relação aos demais $(\mathrm{t}>\mathrm{t}$ crítico $=1,96 ; \mathrm{GL}=\infty$; $\mathrm{p}<0,05$ ) (Tabela 2).

Dentre as 33 espécies amostradas, 15 foram consideradas freqüentes e abundantes em pelo menos um dos pontos de coleta ao longo do período amostral. Na Tabela 3 observa-se a alteração da composição da comunidade ao longo dos $11,1 \mathrm{~km}$ analisados. Várias espécies consideradas frequentes e abundantes se repetem nos pontos de coleta, apesar das localidades apresentarem características fisiográficas distintas. À medida que se direcionam à jusante, onde observa-se a mudança da paisagem, tais espécies tornam-se freqüentes, mas não abundantes, e podem até se tornar ocasionais ou ausentes.

Foi possível observar a inversão das abundâncias das ordens em função da composição fisiográfica de cada localidade. No primeiro ponto de coleta, onde são observados matacões e seixos, ocorre a maior freqüência de Siluriformes $(85,7 \%)$, seguindo de uma diminuição gradativa até o quarto ponto $(40,8 \%)$, onde são mais observados cascalho e areia grossa. $\mathrm{O}$ contrário ocorre em relação às espécies de Characiformes, havendo um aumento gradativo de
Tabela 2. Equitabilidade (J'), Riqueza (R) e número de indivíduos coletados (n) nos pontos de coleta.

Table 2. Equitability (J'), Richness (R) and number of individuals collected in the sampling sites.

\begin{tabular}{cccccc}
\hline Índices & Ponto 1 & Ponto 2 & Ponto 3 & Ponto 4 & Ponto 5 \\
\hline J' & $0,696^{\mathrm{a}}$ & $0,761^{\mathrm{b}}$ & $0,736^{\mathrm{b}}$ & $0,733^{\mathrm{b}}$ & $0,761^{\mathrm{b}}$ \\
$\mathrm{R}$ & 16 & 27 & 24 & 28 & 22 \\
$\mathrm{~N}$ & 1499 & 1334 & 1484 & 1463 & 1251 \\
\hline
\end{tabular}

* a diferença entre as letras demonstra a significância estatística $(\mathrm{p}<0,05)$

sua frequiência, iniciando com apenas $14,3 \%$, atingindo seu máximo em P4 (50,7\%). As tendências gradativas da distribuição das duas ordens não permanecem contínuas até o P5, havendo um aumento da freqüência de Perciformes $(11,1 \%)$, pois esta região sofre grande influência da baía de Antonina devido a sua proximidade ao mar (Figura 7).

A diferença de composição da ictiofauna entre os pontos é evidente ao se realizar a análise de similaridade entre os ambientes (Figura 8). Os pontos 2, 3, 4 e 5 apresentaram similaridade de 59,1\% entre si, sendo que os pontos 4 e 5 apresentaram os maiores valores 
Tabela 3. Diagrama da composição das espécies amostradas.

Table 3. Diagram of the composition of samples species.

\begin{tabular}{|c|c|c|c|c|c|}
\hline Espécies & Ponto 1 & Ponto 2 & Ponto 3 & Ponto 4 & Ponto 5 \\
\hline \multicolumn{6}{|l|}{ Trichomycterus davisi } \\
\hline \multicolumn{6}{|l|}{ Isbrueckerichthys duseni } \\
\hline \multicolumn{6}{|l|}{ Pareiorhaphis splendns } \\
\hline \multicolumn{6}{|l|}{ Rhamdioglanis transfasciatus } \\
\hline \multicolumn{6}{|l|}{ Bryconamericus microcephalus } \\
\hline \multicolumn{6}{|l|}{ Deuterodon langei } \\
\hline \multicolumn{6}{|l|}{ Ancistrus multispinis } \\
\hline \multicolumn{6}{|l|}{ Rineloricaria sp. } \\
\hline \multicolumn{6}{|l|}{ Scleromystax barbatus } \\
\hline \multicolumn{6}{|l|}{ Characidium pterostictum } \\
\hline \multicolumn{6}{|l|}{ Phalloceros caudimaculatus } \\
\hline \multicolumn{6}{|l|}{ Pimelodella pappenheimi } \\
\hline \multicolumn{6}{|l|}{ Characidium lanei } \\
\hline \multicolumn{6}{|l|}{ Mimagoniates microlepis } \\
\hline Awaous tajasica & & & & & \\
\hline
\end{tabular}

Espécies frequentes e abundantes

Espécies frequentes e não abundantes

Espécies ocasionais

Espécies ausentes

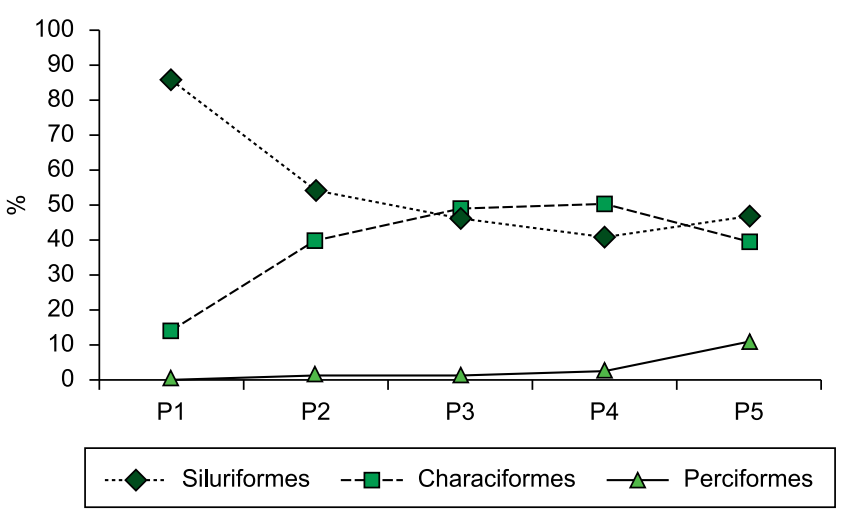

Figura 7. Frequência relativa (\%) de Siluriformes, Characiformes e Perciformes entre os pontos de coleta.

Figure 7. Relative frequency (\%) of Siluriformes, Characiformes and Perciformes along the sampling sites.

(79,6\%). Estes dois últimos pontos também são os mais semelhantes em relação à fisiografia. $\mathrm{O}$ ponto 1 mostrou-se o mais distinto em sua composição ictíica, com apenas $36,5 \%$ de similaridade em relação aos demais, confirmando o resultado já observado na análise de diversidade.

Uma vez caracterizados os pontos, foi possível verificar que há correspondência das espécies em função dos aspectos fisiográficos, sendo que tal correspondência é explicada por $89,73 \%$ da variação dos dados $\left(\chi^{2}=6365,0 ;\right.$ GL $\left.=56 ; p<0,0001\right)$ (Figura 9).

A partir da análise multivariada de correspondência, pôde-se observar que o primeiro eixo demonstra a granulometria, sendo que os escores positivos denotam a ocorrência de matacões e blocos, enquanto os escores negativos demonstram a ocorrência

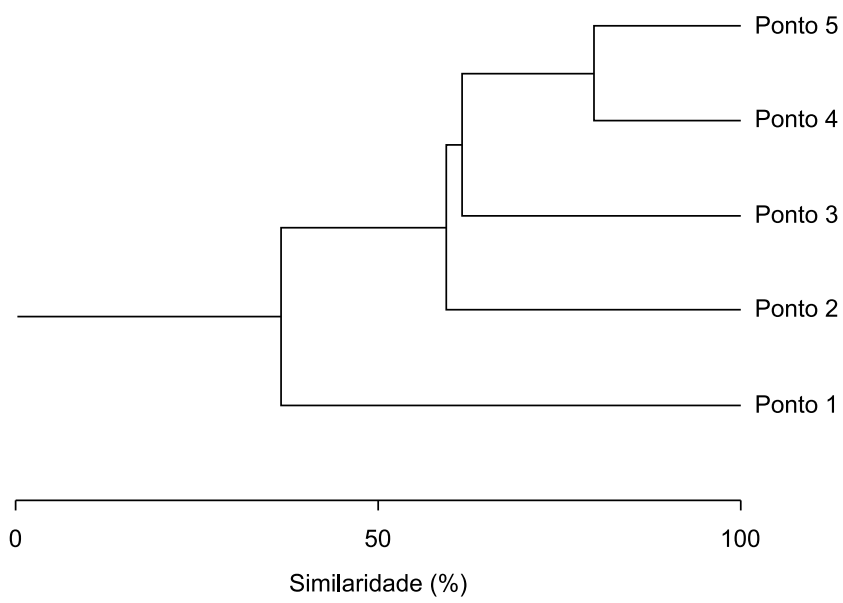

Figura 8. Dendrograma representativo da similaridade dos 5 pontos amostrais em relação à composição de espécies (valor cofenético $=0,89$ ).

Figure 8. Similarity dendrogram of the 5 sampling sites related to the composition of species (cophenetic value $=0.89$ ).

de um substrato rico em seixos, cascalho e areia. O segundo eixo demonstra a localidade dos pontos, considerando-se a variação em relação a altitude dos locais amostrados no Rio do Pinto. Pode-se verificar que à medida que ocorre a diminuição da granulometria ao longo do rio, observa-se a diminuição da frequiência de espécies de Siluriformes, e o aumento de Characiformes. Contudo, em localidades onde há a mistura granulométrica, como por exemplo, matacões e seixos com cascalho e areia grossa, ocorre a formação de hábitats característicos tanto para espécies de corredeira (e.g. Ancistrus multispinnis e Rineloricaria sp.), como para espécies de águas mais calmas (e.g. Phalloceros caudimaculatus e Bryconamericus microcephalus). 


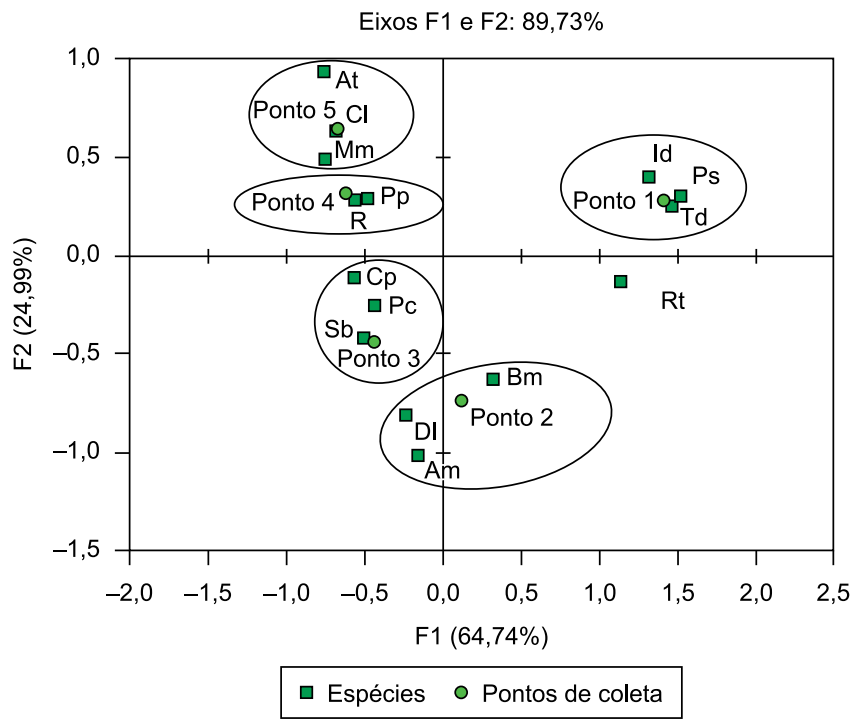

Figura 9. Gráfico de dispersão das duas funções de correspondência. Id - I. duseni, Ps - P. splendens, Td - T. davisi, Bm - B. microcephalus, $\mathrm{Am}-\mathrm{A}$. multispinnis, $\mathrm{Dl}-\mathrm{D}$. langei, $\mathrm{Sb}-\mathrm{S}$. barbatus, $\mathrm{Pc}-\mathrm{P}$. caudimaculatus, $\mathrm{Cp}-\mathrm{C}$. pterostictum, $\mathrm{Pp}-\mathrm{P}$. pappenheimi, $\mathrm{R}-$ Rineloricaria sp., At-A. tajasica, $\mathrm{Cl}-$ C. lanei, $\mathrm{Mm}-\mathrm{M}$. microlepis, $\mathrm{Rt}-\mathrm{R}$. transfasciatus.

Figure 9. Dispersion graph for two functions of the correspondence analysis. Id - I. duseni, Ps - P. splendens, Td - T. davisi, Bm - B. microcephalus, $\mathrm{Am}-\mathrm{A}$. multispinnis, $\mathrm{Dl}-\mathrm{D}$. langei, $\mathrm{Sb}-\mathrm{S}$. barbatus, $\mathrm{Pc}-\mathrm{P}$. caudimaculatus, $\mathrm{Cp}-$ C. pterostictum, $\mathrm{Pp}-\mathrm{P}$. pappenheimi, $\mathrm{R}-$ Rineloricaria $\mathrm{sp}$., At - A. tajasica, $\mathrm{Cl}-\mathrm{C}$. lanei, $\mathrm{Mm}-\mathrm{M}$. microlepis, $\mathrm{Rt}-\mathrm{R}$. transfasciatus.

\section{Discussão}

As variáveis que caracterizam a fisiografia de riachos costeiros influenciam na abundância das diferentes espécies de peixes. Meador \& Goldstein (2003) demonstraram que a composição da ictiofauna se modifica conforme as mudanças ambientais, condições de hábitats e influências antrópicas nos trechos dos rios, como resultado dos processos evolutivos e históricos de adaptações peculiares de cada espécie. As modificações dos hábitats decorrentes da canalização das margens, destruição da vegetação ripária ou barramentos, e deterioração da qualidade da água, exercem uma influência no ecossistema, podendo causar variações na distribuição espaçotemporal na ictiofauna (Waite \& Carpenter 2000). A partir destes fatos, pode-se afirmar que as variáveis físicas de um rio, estando ele em estado natural de conservação ou impactado, apresentam gradiente contínuo de montante à jusante, com as comunidades biológicas ajustando-se por meio da substituição de espécies, com maior eficiência ao consumo de energia. Esta afirmação vai ao encontro do que é preconizado na Teoria do Rio Contínuo (Vannote et al., 1980). Desta maneira, discute-se a mudança da ictiocenose em função da mudança fisiográfica dos locais de coleta.

De acordo com a caracterização fisiográfica de cada um dos locais de coletas, pôde ser verificado que:

a) no ponto 1 prevaleceu um substrato de matacões e blocos, caracterizado por ser um ambiente do tipo corredeira, com correnteza variável de rápida a torrencial. Estas características minimizam o potencial de hábitat para espécies da ordem Characiformes, sendo, contudo, um recurso positivo para a colonização das espécies de Siluriformes, uma vez que estas apresentam diferentes adaptações ecomorfológicas, mas principalmente o achatamento dorso-ventral. Dentre as cinco espécies freqüentes e abundantes observadas nesta localidade, quatro são Siluriformes, sendo que Trichomycterus davisi (Barreto \& Aranha, 2005), I. duseni (Jerep et al., 2006), Pareiorhaphis splendens (Aranha et al. 1998) e Rhamdioglanis transfasciatus (Menezes et al. 2007) habitam em rios de pequeno a médio porte, com correnteza rápida, de água bastante oxigenada e clara, com fundo pedregoso composto de cascalho e matacões.

b) O ponto 2 contém substrato de seixos e cascalho, apresentando sua vazão fluvial regulada pelas precipitações locais, as quais determinam a variação de correnteza moderada a rápida. Pode apresentar episódios de enxurradas, geralmente tendo uma profundidade máxima de $0,5 \mathrm{~m}$. Foram amostradas 27 espécies, considerando-se 10 como freqüentes e abundantes: I. duseni, $P$. splendens, $R$. transfasciatus, A. multispinis, Rineloricaria sp., Scleromystax barbatus, B. microcephalus, Deuterodon langei, $C$. pterostictum e $P$. caudimaculatus. As três primeiras são comuns ao ponto de coleta anterior, uma vez que são observadas características fisiográficas comuns, tais como algumas áreas com matacões, cascalho e correnteza moderada a rápida. Estas características também são ideais para o hábitat das espécies A. multispinis, Rineloricaria sp. e S. barbatus, conforme já observado por Oyakawa et al. (2006) e Menezes et al. (2007). As últimas quatro espécies (B. microcephalus, D. langei, $C$. pterostictum e $P$. caudimaculatus) são Characiformes, as quais foram coletadas principalmente na região marginal, próximas à vegetação. B. microcephalus é característico de riachos de Floresta Atlântica (Mazzoni \& Da Silva 2006), com fundo de areia e pedra e com correnteza rápida, e nadam ativamente na coluna d'água (Oyakawa et al. 2006). D. langei é geralmente coletado em porção inferior da coluna d'água em locais com profundidade de $0,5 \mathrm{~m}$ e na porção média em águas mais profundas, com cerca de 1,5 m (Barreto \& Aranha, 2005). C.pterostictum habita locais de correnteza moderada a torrencial, geralmente associada ao fundo com matacões (Buckup et al., 2000). P. caudimaculatus é encontrado em correnteza lenta a moderada, formando pequenos agrupamentos em águas rasas próximas à margem (Barreto \& Aranha, 2005). A partir de tais observações, constata-se que este trecho apresenta uma fauna ictíica típica, indicando que o mesmo se mostra íntegro em relação, pelo menos, a este grupo faunístico.

c) $\mathrm{O}$ ponto $\mathrm{P} 3$ é semelhante ao ponto P2. Entre as sete espécies freqüentes e abundantes que foram observadas nesta localidade, cinco eram comuns ao ponto P2 (B. microcephalus, A. multispinis, Rineloricaria sp., S. barbatus, C. pterostictum). P. pappenheimi e C. lanei mostraram-se abundantes e frequentes nesta localidade. A observação destas espécies está associada às áreas esparsas com fundo de areia grossa associada ao cascalho e seixos. $P$. pappenheimi é uma espécie de fundo, geralmente encontrada em porções das margens dos rios, onde a velocidade de correnteza é menor (Aranha et al. 1998); no presente estudo, esta espécie foi encontrada formando pequenos cardumes próximos à vegetação ripária, composta principalmente por integrantes da família Poaceae. C. lanei e C. pterostictum foram encontradas nas mesmas localidades, contudo a primeira foi geralmente coletada em locais próximos às margens, onde a correnteza é menor, enquanto a segunda foi coletada em águas com maior correnteza, onde o fundo apresenta-se com mais cascalho e blocos. Tais descrições corroboram com informações descritas por Barreto \& Aranha (2005). 
d) No ponto $\mathrm{P} 4$, com a maior prevalência de areia grossa associada ao cascalho, surge ainda como espécie freqüente e abundante M. microlepis. Esta espécie foi geralmente capturada em locais de pouca profundidade e com velocidade de corrente entre moderada à rápida, assim como o encontrado por Mazzoni \& Iglesias-Rios (2002). Estes mesmos autores afirmam ainda que a presença desta espécie está diretamente correlacionada com a presença de insetos alóctones, que usam a cobertura vegetal como hábitat ideal à reprodução. $P$. pappenheimi e $C$. lanei também se mostraram abundantes e frequentes nesta localidade, assim como no ponto P3, apresentando sua ocorrência condicionada aos mesmos fatores supra citados.

e) No ponto P5, a prevalência de areia grossa associada ao cascalho é ainda maior do que no ponto P4. Esta região apresenta cinco espécies freqüentes e abundantes (Rineloricaria sp., C. pterostictum, P. pappenheimi, C. lanei e A. tajasica), havendo um aumento na ocorrência de Perciformes. Possivelmente, todas estas espécies sejam residentes na presente localidade, sendo características de pequenas bacias fluviais e demonstrando uma fidelidade ambiental (Castro 1999). A única exceção entre estas espécies é A. tajasica, pertencente à família Gobiidae, subfamília Gobioneliinae. É considerada uma espécie não residente, pois provavelmente passa parte do seu ciclo de vida inicial no mar (Lowe-McConnell 1999).

Apesar da evidência de relação entre a distribuição das espécies e a fisiografia dos pontos de coleta, são controversas as opiniões na literatura sobre as variáveis que influenciam diretamente na distribuição das comunidades. Teixeira et al. (2005) afirmaram que as diferenciações na fisiografia ao longo da extensão da bacia do rio Paraíba do Sul não coincidiram com as mudanças de diversidade ictíica. Contudo, desde Shelford (1911), é descrito que a análise fisiográfica do ambiente demarca as espécies aos seus hábitats. Mazzoni \& Iglésias-Rios (2002) demonstraram a forte correlação da cobertura vegetal, vegetação aquática e tipos de substratos em relação à presença ou ausência de espécies, como $M$. microlepis e H. malabaricus, em riachos costeiros. Isto pode ser atribuído a inúmeras variáveis que podem influenciar nas comunidades, mas principalmente às dinâmicas, tais como a granulometria e o regime de fluxo do rio. Vários outros autores demonstram formas de relação da distribuição da ictiofauna com a fisiografia, tais como Belliard et al. (1997) e Oberdorff et al. (2001).

Esta caracterização das espécies em função das localidades de coleta denota a premissa proposta por Cunico \& Agostinho (2006) de que padrões morfológicos estão diretamente relacionados à hidrodinâmica de rios e reservatórios. Estes autores relatam que ambientes com maior hidrodinâmica (lóticos) apresentam espécies com corpo mais achatado ventralmente e com menor compressão lateral, tais como as espécies observadas nos pontos P1 e P2 (I. duseni, $P$. splendens, $R$. transfasciatus, A. multispinis, Rineloricaria $\mathrm{sp.}$, $S$. barbatus). Estas espécies também se caracterizam por apresentarem menores valores do índice de achatamento ventral, ou seja, baixos valores resultantes da razão entre a média de alturas do corpo sobre a altura do corpo (Freire \& Agostinho 2001). Baixos valores deste índice estão associados a espécies bentônicas que se mantêm em ambientes com elevada hidrodinâmica sem precisarem nadar (Cunico \& Agostinho 2006). Contudo, a partir do ponto P2 observam-se menor altitude e sítios com menor velocidade de corrente, caracterizando locais com menor hidrodinâmica quando comparados ao ponto P1. Desta maneira, nestes ambientes são observadas espécies com maior compressão lateral (Watson \& Balon 1984) e maior altura relativa (Gatz Jr. 1979), tais como B. microcephalus, D. langei, P caudimaculatus, M. microlepis e G. brasiliensis. Estas características promovem uma melhor capacidade de deslocamentos verticais.
Contudo, uma vez observada a mudança gradual da distribuição dos peixes, foram encontradas espécies comuns desde o ponto P1 até o ponto P5, tais como $C$. lanei e $C$. pterostictum. Vale ressaltar que em todos os pontos amostrais foram observadas porções do substrato formados por matacões e/ou blocos e seixos, porém com variação em sua frequiência. Desta forma, possibilita a formação de microhábitats para estas espécies. Estas duas espécies pertencem à ordem Characiforme, e apresentam baixos valores relativos ao índice de achatamento ventral, ou seja, pequena média de altura do corpo em relação ao maior valor de altura do corpo (Gatz-Jr. 1979, Freire \& Agostinho, 2001). Além disso, apresentam elevados valores da razão do aspecto da nadadeira peitoral, ou seja, apresentam nadadeiras longas que, segundo Buckup et al. (2000), neste gênero são utilizadas para se apoiar ao substrato rochoso. Mas, C. lanei possivelmente utiliza estas nadadeiras para se apoiar em um substrato predominantemente arenoso, enquanto $C$. petrostictum, as utiliza em um substrato mais rochoso.

Por fim, a alteração da fisiografia nesta microbacia promove a variação da ocupação das espécies de forma gradativa, havendo a alteração do padrão morfológico e ocupacional das espécies, passando de espécies que resistem à elevada força da corrente por meio de adaptações morfológicas, tais como o achatamento dorsoventral característico de espécies de Siluriformes, para espécies que resistem a tal força por meio da compressão lateral do corpo e natação constante na coluna d'água, sendo representados principalmente por Characiformes.

\section{Agradecimentos}

Agradecemos ao apoio dos integrantes do Laboratório de Ecologia de Rios da Universidade Federal do Paraná, em especial ao Dr. José Marcelo Rocha Aranha pelas sugestões críticas. Agradecemos ao Prof. Oswaldo Oyakawa pela identificação de espécies de peixes.

\section{Referências Bibliográficas}

\section{ADDINSOFT. 2009. XLStat 2009. Addinsoft SARL, França.}

ARANHA, J.M.R. 2000. A influência da instabilidade ambiental na composição e estrutura trófica da ictiofauna de dois rios litorâneos. Tese de Doutorado, Universidade Federal de São Carlos, 130p.

ARANHA, J.M.R., TAKEUTI, D.F. \& YOSHIMURA, T.M. 1998. Habitat use and food partioning of the fishes in a coastal stream of Atlantic Forest. Brazil. Rev. Biol. Trop. 46(4):951-959.

ARAÚJO, F.G. 1998. Adaptação do índice de integridade biótica usando a comunidade de peixes para o Rio Paraíba do Sul. Rev. Brasil. Biol. 58(4):547-558.

BARRETO, A.P. \& ARANHA, J.M.R. 2005. Assembléia de peixes de um riacho de Floresta Atlântica: composição e distribuição espacial (Guaraqueçaba, Paraná, Brasil). Acta Sci. Biol. Sci. 27(2):153-160.

BELLIARD, J., BOËT, P. \& TALES, E. 1997. Regional and longitudinal patterns of fish community structure in the Seine River basin, France. Environ. Biol. Fisches 50(2):133-147.

BUCKUP, P.A., ZAMPROGNO, C., VIEIRA, F. \& TEIXEIRA, R.L. 2000. Waterfall climbing in Characidium (Crenuchidae: Characidiinae) from eastern Brazil. Ichthyol. Explor. Freshwaters 11(3):273-278.

CASATTI, L. 2002. Alimentação dos peixes de um riacho do Parque Estadual Morro do Diabo, Bacia do Alto Rio Paraná, Sudeste do Brasil. Biota Neotrop. 2(2):1-14.

CASATTI, L. 2004. Ichthyofauna of two streams (silted and reference) in the upper Paraná River basin, Southeastern Brazil. Braz. J. Biol. 64(4):757-765.

CASATTI, L., LANGEANI, F. \& CASTRO, R.M.C. 2001. Peixes de riacho do Parque Estadual Morro do Diabo, Bacia do Alto rio Paraná, SP. Biota Neotrop. 1(1):1-15. 
CASTRO, R.M.C. 1999. Evolução da ictiofauna de riachos sul-americanos: padrões gerais e possíveis processos causais. In Ecologia de Peixes de Riachos: Estado Atual e Perspectivas (E.P. Caramaschi, R. Mazzoni, C.R.S.F. Bizzeril \& P.R. Perez-Neto, eds.). PPGE-UFRJ, Rio de Janeiro, v. 6, p. 139-155. (Oecologia Brasiliensis).

CUNICO, A.M. \& AGOSTINHO, A.A. 2006. Morphological Patterns of Fish and Their Relationships with Reservoirs Hydrodynamics. Braz. Arch. Biol. Technol. 49(1):125-134.

DAJOZ, R. 2005. Princípios de Ecologia. 7 ed. Artmed, Porto Alegre, $519 \mathrm{p}$.

ESTEVES, K.E. \& LOBÓN-CERVIÁ, J. 2001. Composition and trophic strutucture of a community of a clear water Atlantic rainforest stream in southeastern Brazil. Environ. Biol. Fisches 62: 429-440.

FERREIRA, C.P. \& CASATTI, L. 2006. Influência da estrutura do hábitat sobre a ictiofauna de um riacho em uma micro-bacia de pastagem, São Paulo, Brasil. Rev. Bras. Zool. 23(3):642-651.

FERREIRA, K.M. 2007. Biology and ecomorphology of stream fishes from the rio Mogi Guaçu basin, Southeastern Brazil. Neotrop. Ichthyol. 5(3):311-326.

FREIRE, A.G. \& AGOSTINHO, A.A. 2001. Ecomorfologia de oito espécies da ictiofauna do reservatório de Itaipu (Paraná/Brasil). Acta Limnol. Bras. 13(1):1-9.

FRIBERG, N., DYBKJÆR, J.B., OLAFSSON, J.S., GISLASON, G.M., LARSEN, S.E. \& LAURIDSEN, T.L. 2009. Relationships between structure and function in streams contrasting in temperature. Freshw. Biol. 54:2051-2068.

GATZ Jr., A.J. 1979. Ecological morphology of freshwater stream fishes. Tulane Stud. Zool. Bot. 21(2):91-124.

GRIFFIN, J.N., JENKINS, S.R., GAMFELDT, L., JONES, D., HAWKINS, S.J. \& THOMPSON, R.C., 2009. Spatial heterogeneity increases the importance of species richness for an ecosystem process. Oikos 118:1335-1342.

GROSSMAN, G.D., MOYLE, P.B. \& WHITAKER Jr., J.O. 1982. Stochasticity in structure and functional characteristics of an Indiana stream fish assemblage: a test of community theory. Am. Nat. 120:423-454.

HAIR, J.F., ANDERSON, R.E., TATHAM, R.L. \& BLACK, W.C. 1998. Multivariate data analysis. 5 ed. Prentice Hall, New Jersey, 768p.

JEREP, F.C., SHIBATTA, O.A., PEREIRA, E.H.L. \& OYAKAWA, O.T 2006. Two new species of Isbrueckerichthys Derijst, 1996 (Siluriformes: Loricariidae) from the rio Paranapanema basin, Brazil. Zootaxa 1372:53-68.

LEMES, E.M. \& GARUTTI, V. 2002. Ecologia da ictiofauna de um córrego de cabeceira da bacia do Alto Rio Paraná, Brasil. Iheringia 92(3):69-78.

LOEBMANN, D. \& VIEIRA, J.P. 2005. Composição e abundancia dos peixes do Parque Nacional da Lagoa do Peixe, Rio Grande do Sul, Brasil e comentários sobre a fauna acompanhante de crustáceos decápodos. Atlântica 27(2):131-137.

LOWE-MCCONNELL, R.H. 1999. Estudos ecológicos de comunidades de peixes tropicais. EDUSP, São Paulo, 536p.

MATHEWS, W.J. 1986. Fish faunal structure in an Ozark stream: stability, persistence and a catastrophic flood. Copeia 1986(2):388-397.

MAZZONI, R. \& DA SILVA, A.P.F. 2006. Aspectos da história de vida de Bryconamericus microcephalus (Miranda Ribeiro) (Characiformes, Characidae) de um riacho costeiro de Mata Atlântica, Ilha Grande, Rio de Janeiro, Brasil. Rev. Bras. Zool. 23(1):228-233.
MAZZONI, R. \& IGLÉSIAS-RIOS, R. 2002. Distribution pattern of two fish species in a coastal stream in southeast Brazil. Braz. J. Biol. 62(1):171-178

MEADOR, M.R. \& GOLDSTEIN, R.M. 2003. Assessing water quality at large geographic scales: relations among land use, water physicochemistry, riparian condition, and fish community structure. Environ. Manag. 31(4):504-517.

MENEZES, M.S., TAKEUTI, D.F., ARANHA, J.M.R. \& VERANI, J.R. 2000. Desenvolvimento Gonadal de machos e fêmeas de Pseudotothyris obtusa (Ribeiro,1911) (Loricariidae, Hypoptopomatinae). Acta Biol. Par. 29(1-4):89-100.

MENEZES, N.A., HEITZMAN, S.H., OYAKAWA, O.T., DE LIMA, F.C.T., CASTRO, R.M.C. \& WEITZMAN, M.J. 2007. Peixes de água doce da Mata Atlântica. Museu de Zoologia da Universidade de São Paulo, São Paulo, 407p.

OBERDORFF, T., PONT, D., HUGUENY, B. \& CHESSEL, D. 2001. A probabilistic model characterizing fish assemblages of French rivers: a framework for environmental assessment. Freshw. Biol. 46(3):399-415.

OYAKAWA, O.T., AKAMA, A., MAUTARI, K.M. \& NOLASCO, J.C. 2006. Peixes de Riachos da Mata Atlântica. Ed. Neotropica, São Paulo, 201p.

SHELFORD, V.E. 1911. Ecological succession: stream fishes and the method of physiographic analysis. Bio. Bull. 21:9-35.

SILVEIRA, M.P. 2004. Aplicação do Biomonitoramento para Avaliação da Qualidade da Água em Rios. Embrapa Meio Ambiente. 68 p. (Documentos 36).

TEIXEIRA, W., TOLEDO, M.C.M., FAIRCHILD, T.R. \& TAIOLI, F. 2000. Decifrando a Terra. Oficina dos Textos, São Paulo, 568p.

TEIXEIRA, T.P., PINTO, B.C.T., TERRA, B.F., ESTILIANO, E.O., GARCIA, D. \& ARAÚJO, F.G., 2005. Diversidade das assembléias de peixes nas quatro unidades geográficas do rio Paraíba do Sul. Iheringia, Sér. Zool., 95(4): 347-357.

TEJERINA-GARRO, F.L., MALDONADO, M., IBAÑEZ, C., PONT, D., ROSET, N. \& OBERDORFF, T. 2005. Effects of naturaland anthropogenic environmental changes on riverine fish assemblages: a framework for ecological assessment of rivers. Braz. Arch. Biol. Tech. 48(1):91-108.

VANNOTE, R.L., MINSHALL, G.W., CUMMINS, K.W., SEDELL, J.R. \& CUSHING, C.E. 1980. The River Continuum Concept. Can. J. Fish. Aquat. Sci. 37(1):130-137.

WAITE, I.R. \& CARPENTER, K.D. 2000. Associations among fish assemblage structure and environmental variables in Willamette Basin streams, Oregon. Trans. Am. Fish. Soc. 129(3):754-770.

WATSON, D.J. \& BALON, E.K., 1984. Ecomorphological analysis of fish taxocenes in rainforest streams of northern Borneo. J. Fish Biol. 25(3):371-384.

YANT, P.R., KARR, J.R. \& ANGERMEIER, P.L. 1984. Stochasticity in stream fish communities: an alternative interpretation. Am. Nat. 124(4):573-582.

ZAR, J.H. 1998. Biostatistical Analysis. 4 ed. Prentice-Hall, Englewood Cliffs, 929p.

Recebido em 26/10/09

Versão reformulada recebida em 04/02/10

Publicado em 22/04/10 